\title{
Conocimientos, creencias y actitudes ante la violencia en el noviazgo en estudiantes mujeres que cursan el último año de bachiller en una escuela oficial en la ciudad de Panamá
}

\section{Montserrat Muñoz,"*}

${ }^{1}$ Estudiante, Escuela de Psicología, Universidad Santa María la Antigua, Apartado Postal 0819-08550, Panamá, Rep. de Panamá

*Autor para correspondencia. Email: mdanielamunoz@hotmail.com

Recibido: 28 de febrero de 2014

Aceptado: 15 de abril de 2014

\begin{abstract}
In Panama, we found few studies on teen dating violence, so nowadays it is difficult to establish its severity and prevalence in adolescent couples. The present study aims to characterize the beliefs and attitudes of a sample of 33 Panamanian students about violent behavior in relationships. A questionnaire designed for the purpose of research that measures four dimensions was applied. The dimensions where: attitudes toward relationships, physical violence, respect and communication (belonging to psychological) and self-esteem.

The main factors associated with violence found in the sample of adolescents are the abilities to communicate, accompanied by myths and stereotypes aimed to justify violence in the relationship.
\end{abstract}

Keywords: Adolescent romantic relationship, Dating violence, Physical violence, Psychological violence, Beliefs. 
Invest. pens. crit.

Vol. 2, No. 3, enero-abril 2014.

pp. 26-34

\section{Resumen}

En Panamá, encontramos escasos estudios sobre la violencia en el noviazgo adolescente, por lo tanto es difícil establecer su gravedad y prevalencia en las parejas adolescentes hoy en día. El presente estudio tiene como objetivo caracterizar las creencias y actitudes de una muestra de 33 estudiantes panameñas en cuando al comportamiento violento en las relaciones de pareja. Se aplicó un cuestionario diseñado para los fines de la investigación que mide cuatro dimensiones: actitudes hacia las relaciones de pareja, violencia física, respeto y comunicación (pertenecientes a la violencia psicológica) y autoestima.

Los principales factores asociados a la violencia que se encuentran en la muestra de adolescentes son la capacidad para comunicarse, acompañada de mitos y estereotipos dirigidos a justificar la violencia dentro de la relación de pareja.

Palabras claves: Noviazgo adolescente, Violencia en el noviazgo, Violencia física, Violencia psicológica, Creencias.

\section{Introducción}

La violencia es un comportamiento aprendido a lo largo de la vida del ser humano (Baron y Byrn, 2005), y se transmite generacionalmente a través de creencias que surgen en el seno de las familias (Losso, 2001). El propósito de este estudio es poder investigar sobre la presencia de la misma, junto con los factores que la acompañan en parejas de adolescentes en Panamá.

La Organización Mundial de la Salud _-OMS - (2002) define la violencia como el uso intencional de la fuerza o el poder físico, de hecho o como amenaza, contra uno mismo, otra persona o un grupo o comunidad, que cause o tenga muchas probabilidades de causar lesiones, muerte, daños psicológicos, trastornos del desarrollo o privaciones. Dentro de la clasificación de la violencia, la OMS (2002) identifica tres tipos: la violencia autoinfligida (comportamiento suicida y autolesiones), la violencia interpersonal (violencia familiar, que incluye menores, pareja y ancianos; así como violencia entre personas sin parentesco) y la violencia colectiva (social, política y económica).

Considerando los estudios realizados por la OMS, podemos decir que la violencia está asociada a una serie de creencia, mitos y tradiciones las cuales van alimentando las diferencias y estereotipos de género. Aunado a esto, el creciente aumento de la violencia en el ámbito social, familiar y cultural ha influido en la percepción haciendo de esta un acto aceptado y común en la vida de los adolescentes y sus familias. La presente investigación se enmarca en la violencia de tipo interpersonal, que incluye la violencia en la pareja (OMS, 2002).

Es necesario conocer cuál es la posición de la adolescencia panameña ante las relaciones de pareja, ya que estas serán la base para la construcción futura de la familia. Con motivo de esta problemática, James Makepeace, en la década de los ochenta, realiza la primera investigación 
Invest. pens. crit.

Vol. 2, No. 3, enero-abril 2014.

pp. 26-34

para recolectar datos sobre la violencia presentada en el noviazgo adolescente. Esta primera publicación llamada "Courtship violence among college students" (Makepeace, 1981) logra establecer datos fundamentales, logrando así que tome una importancia primordial no solo para su estudio, sino para su prevención y orientación de la misma en dicha población. Posteriormente se han realizado investigaciones y programas de prevención contra la violencia en parejas adolescentes en países como en México (Fize, 2001), España (Perinat, 2003; Yuberto, 2007), Chile (Cárdenas, 1999), entre otros.

La información estadística del Instituto Nacional de Estadística y Censo de la Contraloría General de la República de Panamá nos indican que durante el año 2010 (Baron y Byrne, 2005; INEC, 2011, 2012; Valverde, 2013), 94.6 hombres y 16.6 mujeres por cada cien mil habitantes, murieron por causas violentas. Durante el año 2011 la cantidad de muertes por violencia disminuyeron a 13.7 las mujeres y a 88 los hombres por cada cien mil habitantes. Por último durante el año 2012 se registraron 712 muertes entre hombre y mujeres, las cuales se dieron por agresiones o actos violentos, esto nos indica que la violencia es la segunda causa de muerte en Panamá.

Estudios previos realizados en la ciudad de Panamá sobre el tema, indican que el 77\% de la población femenina de jóvenes entre los 18 y 25 años han presentado indicios de violencia en sus relaciones (Barrios, 2007). Por otro lado, publicaciones realizadas en México sobre estudios en Panamá y en países como Belice, Honduras, Costa Rica y Nicaragua, indican que Panamá se encuentra en el cuarto lugar en cuanto a porcentaje de parejas violentas registradas durante el estudio (Pantelides y Manzelli, 2005).

En Panamá, existen investigaciones sobre la violencia en adolescentes (Caballero, 2009; MEDUCA, 2013; Ortega, Ortega y Sánchez, 2008) mas éstas fueron dirigidas a detectar la violencia en general. Dirigiéndonos más al tema de interés, Alejandra Edith Pantelides y Hernán Manzelli (2005), realizan una investigación en países centroamericanos sobre la violencia en el noviazgo, la cual refleja datos importantes sobre su prevalencia, tomando en cuenta la edad, sexo, nivel de estudios, entre otros. El estudio evidenció datos contundentes con respecto a la prevalencia de la violencia dentro del noviazgo, sobre todo en hombres entre los 25 y 35 años de edad, los cuales no terminaron el nivel básico de escolaridad. Al mismo tiempo, el estudio realizado por Delia Barrios (2007) en la ciudad de Panamá, mostró que aproximadamente un $20 \%$ de las estudiantes se encontraban en una relación de abuso, y un 10\% en una relación de abuso severo. Encontramos aquí, que aproximadamente un 30\% de la población de estudiantes en Panamá se encuentran en una relación con algún grado de violencia identificada.

Una revisión de las tesis desarrolladas en Panamá durante los años 2012 a 2014; revela la escasez de datos nacionales sobre la violencia en el noviazgo adolescente. Por ello, esta investigación se posiciona como una aproximación al estudio de esta problemática. 
Invest. pens. crit.

Vol. 2, No. 3, enero-abril 2014.

pp. 26-34

Aún se necesita ampliar la información sobre el tema en cuestión, es fundamental que el profesional de la salud mental recuerde la relevancia de una intervención temprana ante lo que hoy en día es un trastorno social muy importante en nuestro país. Dicha ampliación sobre el tema y recolección de datos sobre el mismo podrá, posteriormente, ser utilizada para crear un programa de intervención que vaya de acorde con la cultura y creencias de nuestro país.

El presente artículo discute los resultados obtenidos de una investigación realizada en el año 2013 como parte del curso Investigación Psicológica I de la Licenciatura en Psicología. El objetivo de dicha investigación plantea poder describir los tipos de violencia que pueden presentarse en el noviazgo adolescente en la ciudad de Panamá. Se pretende caracterizar las creencias y actitudes frente a la violencia en el noviazgo de adolescentes panameños, para así poder describir los tipos de violencia que pueden existir en el noviazgo adolescente, junto con las creencias y actitudes asociadas con la presencia del mismo en la actualidad.

\section{Materiales y métodos}

El presente estudio corresponde a una investigación no-experimental con alcance descriptivo y observación de corte transversal en un solo grupo. El objetivo primordial de esta investigación es poder recolectar datos sobre el conocimiento de la violencia en el noviazgo en las adolescentes panameños que estén cursando el último año de la escuela secundaria en el distrito de San Miguelito en Panamá. Para lograr este objetivo se realizó una encuesta de escala tipo Likert con cinco opciones de respuestas inédita, validada y estructurada específicamente para esta investigación, la cual pretende medir la violencia en el noviazgo en adolescentes.

\section{$\underline{\text { Participantes }}$}

Se utilizó una muestra no probabilística conformada por 33 estudiantes de sexo femenino, matriculadas en el doceavo grado de una escuela oficial de Panamá ubicada en el distrito de San Miguelito. La selección de la muestra se realizó mediante técnica de muestreo por conveniencia, utilizando los grupos asignados por la Dirección del plantel evaluado. Esta población coresponde a un estrato socio-económico bajo ya que residen mayormente en zonas de alto riesgo en Panamá y se consideran adolescentes en riegos por la calidad social del lugar en donde viven. El rango de edad varía entre 17 y 18 años.

\section{$\underline{\text { Medidas }}$}

Se diseñó un cuestionario para medir cinco variables, las cuales están dirigidas a evaluar la presencia de violencia en el noviazgo de la muestra estudiada. El cuestionario se elaboró basándose en reactivos con escala de respuesta tipo Likert de 5 opciones las cuales correspondían a: totalmente de acuerdo, de acuerdo, indeciso, en desacuerdo y totalmente en desacuerdo. Se estipuló un puntaje que varió de 1 a 5 dependiendo del contenido de las preguntas. Para efectos del estudio, la relación del puntaje fue directamente proporcional al grado de violencia presente en la relación de pareja, dicho esto, mientras mayor fue el puntaje obtenido en las encuestas, se 
Invest. pens. crit.

Vol. 2, No. 3, enero-abril 2014.

pp. 26-34

consideró que el sujeto poseía mayor cantidad de características presentes en las variables que apoyaban la violencia en la relación de pareja y viceversa.

Dentro de las áreas medidas se consideraron: actitudes hacia las relaciones de pareja, violencia física, respeto y comunicación (pertenecientes a la violencia psicológica) y autoestima. La dimensión de actitudes contiene 10 preguntas (rango de puntuaciones posibles 10-50) que evalúan las creencias, mitos y estereotipos que pueden tener los adolescentes son respecto a la relación de pareja. Dentro de violencia física, se incluyeron ocho afirmaciones (rango de puntuaciones posibles 8-40), las cuales buscan medir si el sujeto ha recibido algún tipo de agresión física de parte de su pareja y la gravedad de la misma. Con respecto al área de respeto, se plantearon ocho afirmaciones (rango de puntuaciones posibles 8-40) que evaluaran aspectos positivos y negativos sobre la forma en que la pareja se dirige hacia la persona, en términos de insultos, gritos, burlas y humillaciones en diferentes contextos. Para medir autoestima, se implementaron diez reactivos (rango de puntuaciones posibles 10-50), los cuales califican el grado de intereses y cosas positivas que tienen los adolescentes en sus vidas. Por último, la dimensión de comunicación, está conformada por siete reactivos (rango de puntuaciones posibles 7-35) que van dirigidos a medir la capacidad de los adolescentes para comunicar sus pensamientos, sueños y deseos a su pareja.

Se estimó la validez de contenido de la escala aplicando un método basado en el propuesto por Lynn (1986). Se conformó una muestra intencional de 5 expertos en el área de la salud mental con especialidades en violencia, problemas de conducta en adolescencia, salud mental aplicada al área social, psicología clínica de niños y adolescentes. Con base a la evaluación de los jueces expertos se eliminaron 10 reactivos y se modificaron 20 .

\section{Procedimiento}

Se reunió al grupo de estudiantes y se les explicó los objetivos del presente estudio. Se aplicó el cuestionario previamente validado por jueces a las jóvenes que accedieron voluntariamente a participar del estudio. Los cuestionarios completados fueron calificados sumando las puntuaciones obtenidas en cada una de las dimensiones. Se utilizó un análisis estadístico donde se tomaron en cuenta las descripciones estadísticas de mediana y rango intercuartil porque las

variables presentaban una distribución unimodal sesgada. Además, utilizamos frecuencias y porcentajes.

\section{Resultados}

Pudimos encontrar que los resultados de las dimensiones de actitudes, violencia física y respeto muestran una distribución unimodal sesgada.

Dentro de la dimensión de Actitudes, por ser una distribución unimodal, tomamos en cuenta la mediana que presenta un valor de 20 el puntaje máximo es de 38 y el mínimo de 12 con un rango intercuartil de 9.25. Esto nos indica que la mayoría de estudiantes tienen actitudes que van en 
Invest. pens. crit.

Vol. 2, No. 3, enero-abril 2014.

pp. 26-34

contra de la violencia en el noviazgo, ya que la mediana es más cercana al puntaje mínimo anteriormente expuesto.

Por otro lado, en violencia física se presenta una mediana de 12 con un rango de 9 como mínimo y 33 como máximo con un rango intercuartil de 7.75, c la mayoría de las estudiantes no han sufrido violencia física en sus relaciones.

La dimensión de respeto nos muestra una mediana del 13.5 con un rango de 10 y 20 con un rango intercuartil de 5.5 esto demuestra que la mayoría de las estudiantes mantienen respeto dentro de su relación de noviazgo.

En las dimensiones de comunicación y autoestima, el análisis de confiabilidad revela una consistencia interna inter-ítem inadecuada por lo que se realizó el análisis pregunta por pregunta. Los resultados se muestran a continuación.

En la dimensión de comunicación el 61.9\% de las estudiantes mostraron actitudes que iban de acuerdo con mentir a su pareja para evitar problemas y un $9.5 \%$ mostraron actitudes ambivalentes en cuanto al tema (indecisas). El 52.7\% de las estudiantes se mostraron de acuerdo cuando se les preguntó si su novio no les permite que lloren y solo un 5.3\% mostraron ambivalencia ante esto. En cuanto a la comunicación el $28.6 \%$ se sienten incómodas comunicando sus metas a su pareja y un $23.8 \%$ no pueden comunicar como se sienten.

Por parte de la pareja a la hora de comunicarse un 9.5\% de estudiantes dicen que no se les permite decir sus opiniones a sus parejas y un $19 \%$ se presenta indeciso ante esto. Un $28.6 \%$ manifiesta que su pareja se marcha sin decirle cuando está molesto y un 33.4\% indican que su pareja les deja de hablar cuando hay un problema.

En cuanto a autoestima los datos recolectados muestran que el 30\% de las estudiantes no se sienten capaces de abandonar su relación cuando lo desean y un $25 \%$ se muestran indecisas. Referente a las creencias el $100 \%$ de las estudiantes creen que merecen amor y respeto en su relación, al mismo tiempo todas se sienten capaces de elegir a su pareja romántica.

Cuando se realizaron preguntas que iban dirigidas directamente a violencia explícita el $9.6 \%$ de las estudiantes manifestaron saber que se encuentran en una relación violenta y un $9.5 \%$ no pudieron determinar si estaban o no en una relación violenta.

Un 22.8\% de las adolescentes afirmaron que su pareja las ha amenazado con dejarlas si no hacen lo que la pareja quiere y un $4.8 \%$ afirman haber recibido chantajes de parte de la misma. Un 9.6\% de la población ha sufrido burlas y humillaciones por parte de su pareja en cuanto a la apariencia física. Por último el 95\% de las estudiantes afirman tener cosas en su vida que mantienen su interés y solo un $5 \%$ se muestra insegura en cuanto a este reactivo. 
Invest. pens. crit.

Vol. 2, No. 3, enero-abril 2014.

pp. 26-34

\section{Discusión}

En el presente estudio las variables no mostraron el grado de violencia esperado, según datos de un estudio previo que señala presencia de indicios de relaciones violentas en el $50 \%$ de los jóvenes y participación en una relación violenta en aproximadamente un 30\% (Barrios, 2007). En contraste, nuestra investigación revela que la mayoría de las estudiantes participantes presentan actitudes, actos de respeto y comunicación que no apoyan la violencia. El mayor déficit se encuentra en las herramientas para comunicarse y en algunas actitudes específicas. Esta diferencia puede deberse a las limitaciones de la muestra obtenida: número reducido de participantes que consistió solo mujeres procedentes de estratos socio-económicos medio bajo con un rango de edad reducido y que no fueron seleccionadas de manera aleatoria. Al mismo tiempo, estas limitaciones están relacionadas con las posibles resistencias a contestar temas sensitivos que pueden aparecer en cuestionarios de auto-reporte.

Los datos obtenidos sobre las habilidades de comunicación y autoestima reflejan que las adolescentes que conformaron la muestra de estudio tienen un déficit importante en las habilidades básicas para comunicarse con la pareja. Específicamente, se hallaron dificultades en habilidades para comunicar pensamientos, emociones, inquietudes, metas y sueños a su pareja. Es posible que esta dificultad en la comunicación de afectos sea aprendida desde los patrones culturales encontrados en nuestra sociedad (Martínez, Leis \& Terán, 2012). Por otro lado, un porcentaje alto de estudiantes manifestaron sufrir algún tipo de control o abuso psicológico de las cuales muchas de estas actitudes fueron confundidas como demostraciones de amor por su pareja. Estas creencias pueden ser reforzadas por el aprendizaje intergeneracional de las expectativas de rol encontradas en nuestra sociedad (Barrios, 2007). Tomando en cuenta este resultado se considera que los programas de intervención deben trabajar sobre acciones dirigidas a la modificación de estas creencias.

Nuestros datos indican que la violencia psicológica es más común que la física en las relaciones de pareja adolescentes e, incluso, parece existir mayor tolerancia frente a esta en vista de que muchas adolescentes no consideraban el abuso psicológico y emocional como violencia.

El presente estudio apoya la necesidad de promover nuevas investigaciones que permitan evaluar la percepción de los temas aquí analizados desde el punto de vista de los adolescentes masculinos. También se requiere ampliar el estudio a muestras más representativas de diferentes entornos sociales, explorando las dificultades de carácter social que pueden estar implicadas en la violencia relacionada con el noviazgo adolescente. Esta línea de investigación permitirá anticiparnos a las repercusiones del problema a largo plazo y ayudará al establecimiento de medidas de prevención e intervención acordes a las necesidades de la población panameña. 
Invest. pens. crit.

Vol. 2, No. 3, enero-abril 2014.

pp. 26-34

\section{Agradecimiento}

Agradezco al Profesor Rubén Díaz, ya que sin su apoyo y guía no hubiera sido posible la construcción de éste artículo.

\section{Referencias}

Barilari S. (2009). Equipo de capacitación en el abordaje de problemáticas sociales. Recuperado el 2012, de EcapSocial: http://www.ecapsocial.com.ar/files/Noviazgos_Violentos.pdf

Baron, R., \& Byrne, D. (2005). Psicología Social. Barcelona, España: Pearson.

Barrios, D. B. (2007). Estereotipos de género y violencia intrafamiliar en estudiantes varones entre 18 a 25 años y su relación con las manifestaciones de violencia en el noviazgo en estudiantes mujeres entre 18 a 25 años. (Tesis de maestría), Universidad de Panamá , Centro Regional Universitario de Azuero, Azuero.

Baztán, Á. A. (1994). Psicología de la Adolescencia. Barcelona, España: Boixarei Universitaria.

Caballero, D. (2009). La violencia en las escuelas. Evaluación del clima del aula en las escuelas primarias públicas del Corregimiento de San Felipe. (Tesis doctoral). Universidad Especializada de las Américas, Panamá.

Cárdenas, E. J. (1999). Violencia en la pareja: Intervenciones para la paz desde la paz. Barcelona, España: Ediciones Juan Granica S.A.

Fize, M. (2001). ¿Adolescencia en crisis?: Por el derecho al reconocimiento social. México DF, México: siglo XXI editores S.A.

INEC. (2012). Estadísticas Vitales, Volumen III - Defunciones: Año 2012. Instituto Nacional de Estadística y Censo, Contraloría General de la República, Panamá.

INEC. (2011). Estadísticas Vitales, Volumen III - Defunciones: Año 2011. Instituto Nacional de Estadística y Censo, Contraloría General de la República, Panamá.

Hormachea, D. (2005). Noviazgo con propósito. Nashville, TN, E.U.A.: Caribe Inc.

Losso, R. (2001). Psicoanálisis de la familia: recorridos teórico-clínicos. Buenos Aires; México: Grupo Editorial Lumen.

Lynn, M. R. (1986). Determination and quantification of content validity. Nursing Research, $35(6), 382-385$.

Martínez, E. G. Noviazgo entre adolescentes. México: Instituto Aguascalentense de las Mujeres. 
Invest. pens. crit.

Vol. 2, No. 3, enero-abril 2014.

pp. 26-34

Martínez, L. M. (18 de abril de 2008). Representaciones sociales del noviazgo en adolescentes escolarizados de estratos socioeconómicos bajo, medio y alto de la ciudad de Bogotá. Bogotá, Bogotá, Colombia

Martínez, S., Leis, M., y Terán, L. A. (2012). Estudio de patrones de convivencia escolar en Panamá (p. 150). Panamá: Centro de Estudio y Acción Social Panameño.

MEDUCA. (2013). Estadística educativa: Final del año escolar 2012. Panamá: Ministerio de Educación.

Moreno, C. I. (2007). Crecer en el noviazgo. Lima, Perú: Paulinas.

Ortega, R., Ortega, F., \& Sánchez, V. (2008). Violencia sexual entre compañeros y violencia en parejas adolescentes. International Journal of Psychology and Psychological Therapy, $8(1), 63-72$.

Pantelides, E.; Manzelli, H. (2005). Violencia en la pareja. Evidencias a partir de encuestas a hombres centroamericanos. Papeles de Población, julio-septiembre, 247-270.

Perinat, A. (2003). Los adolescentes del siglo XXI. Barcelona, España: UOC.

Valverde, Z. (2013). Situación de salud de Panamá. Panamá: Ministerio de Salud.

Velásquez Rivera G. (2011). La violencia durante el noviazgo en adolescentes. Ciencia y Cultura Elementos, 18 (82), 39.

Yubero Imenéz, S., Blanco Abarca, A., \& Arrañaga Rubio, E. (2007). Convivir con la violencia. Cuenca, España: Servicio de publicación de la Universidad de Castilla.

Zacarés, A. (2005). La violencia de género explicada a mi hijo. Valencia: Carena. 